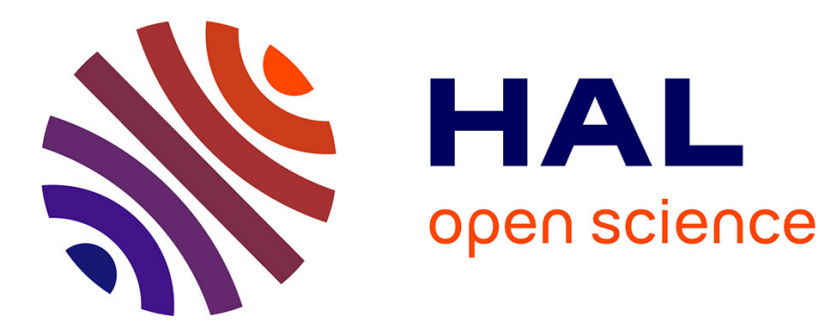

\title{
Experimental observation of a traveling plasma grating formed by two crossing filaments in gases
}

\author{
Magali Durand, Yi Liu, Benjamin Forestier, Aurélien Houard, André
}

Mysyrowicz

\section{- To cite this version:}

Magali Durand, Yi Liu, Benjamin Forestier, Aurélien Houard, André Mysyrowicz. Experimental observation of a traveling plasma grating formed by two crossing filaments in gases. Applied Physics Letters, 2011, 98 (12), pp.121110. 10.1063/1.3568888 . hal-00852001

\section{HAL Id: hal-00852001 \\ https://hal-polytechnique.archives-ouvertes.fr/hal-00852001}

Submitted on 19 Aug 2013

HAL is a multi-disciplinary open access archive for the deposit and dissemination of scientific research documents, whether they are published or not. The documents may come from teaching and research institutions in France or abroad, or from public or private research centers.
L'archive ouverte pluridisciplinaire HAL, est destinée au dépôt et à la diffusion de documents scientifiques de niveau recherche, publiés ou non, émanant des établissements d'enseignement et de recherche français ou étrangers, des laboratoires publics ou privés. 


\title{
Experimental observation of a traveling plasma grating formed by two crossing filaments in gases
}

\author{
Magali Durand ${ }^{1,2}$, Yi Liu ${ }^{1}$, Benjamin Forestier ${ }^{1}$, Aurélien Houard ${ }^{1}$, and André Mysyrowicz ${ }^{1, *}$ \\ ${ }^{1}$ Laboratoire d'Optique Appliquée, ENSTA ParisTech, Ecole Polytechnique, CNRS, 91761 \\ Palaiseau, France \\ ${ }^{2}$ ONERA, Chemin de la Hunière, 91761 Palaiseau, France
}

\begin{abstract}
The spatial motion and effective duration of a traveling plasma grating formed by two interfering femtosecond laser filaments in gases is characterized by its Doppler effect imparted on a probe pulse. The shift velocity determined experimentally agrees with the theoretical calculations.
\end{abstract}

Nonlinear filamentation propagation of intense femtosecond $\left(10^{-15} \mathrm{~s}\right)$ pulses in transparent materials, such as ambient air and water, gives rise to many spectacular phenomena, including supercontinuum generation, formation of long plasma channels, pulse selfcompression and terahertz radiation [1,2]. This nonlinear propagation process has attracted many research interests. The reason is not only the rich physics involved, but also its various potential applications, such as remote sensing of atmospheric pollution [3], pulse compression down to few cycle regime [4], triggering and guiding of electric discharge [5], remote $\mathrm{THz}$ pulse generation [6], to name a few. In the last few years, the interaction of two filaments in gases and solids has triggered new research interests [713]. It was found that the interaction of two filaments can enhance the yield of third order harmonic from a filament in air by 2 orders of magnitude [8]. A stationary plasma grating formed in ambient air, at the overlap region of two laser filaments of same frequencies, can be useful for high intensity laser applications and possibly the formation of lattice solitons [9-11], while a traveling plasma grating was recently proposed by Liu et al [14] to explain the efficient energy exchange between two crossing femtosecond filaments of different frequencies. Up to now, the presence of this traveling plasma grating has not yet been confirmed experimentally. Furthermore, its basic properties such as its traveling velocity and effective duration are still largely unknown.

In this letter, we first capture the spatial profile of the traveling plasma grating. It is found that the plasma grating fringes do not wash out in a time-integrated picture even though they travel with a velocity as high as $7.8 \%$ of the light velocity, as will be shown below. Next, the traveling nature of this plasma grating is demonstrated by the Doppler shift that it imparts on a $400 \mathrm{~nm}$ probe pulse. The velocity of this grating derived from the measurement agrees with the calculation.

In the experiment, a commercial chirped-pulse-amplification (CPA) laser system is employed (Alpha 100, Thales laser). This laser delivers $15 \mathrm{~mJ}, 40 \mathrm{fs}$ laser pulses with central wavelength at $800 \mathrm{~nm}$ at a repetition rate of $100 \mathrm{~Hz}$. The laser beam with $15 \mathrm{~mm}$ diameter is first splitted by a 10/90 beam splitter, with the $10 \%$ beam used as a probe pulse. The $90 \%$ pump beam is further split in two beams with a 50/50 beam splitter. The two pump pulses are focused by two identical convex lenses of $f=1000 \mathrm{~mm}$ in a gas

\footnotetext{
*Corresponding author: andre.mysyrowicz@ensta-paristech.fr
} 
chamber to form two filaments in different gases. The two filaments cross each other with an angle $\varphi$. For a description of the setup see Fig. 1. The time delay between the two pump pulses $\tau_{d}$, and the time delay between the probe and the grating formed by the two pumps $\tau_{p r-g}$, can be varied individually with two optical delay lines. The polarization of the pump pulses is set to be vertical. A type I BBO crystal with a thickness of $100 \mu \mathrm{m}$ is inserted into the probe beam path to generate a probe pulse at $400 \mathrm{~nm}$. The $400 \mathrm{~nm}$ probe pulse obtained after the $\mathrm{BBO}$ crystal is combined with pump 2 by a dichroic mirror. The $400 \mathrm{~nm}$ probe propagates in the same direction as the pump 2 and is expected to be diffracted in the direction of pump 1 in the presence of a plasma grating, since it satisfies the Bragg condition, as discussed later. For the detection of the $400 \mathrm{~nm}$ probe in the direction of pulse 1, color filters (BG 40 and BG 39) are used to attenuate the strong component of the laser spectrum around $800 \mathrm{~nm}$. The diffracted $400 \mathrm{~nm}$ beam is finally focused by a $100 \mathrm{~mm}$ focal lens and coupled into a fiber spectrometer.

In the experiment when the two pump pulses interact without frequency difference, a stationary interference pattern is formed and a resulting static electron plasma grating is generated [10-11]. The period of this interference pattern is given by $\Lambda=\lambda /(2 \sin (\varphi / 2))$, where $\lambda$ and $\varphi$ are respectively the wavelength of the incident pump pulses and the crossing angle. Since the laser intensity is clamped to a value around $5 \times 10^{13} \mathrm{~W} / \mathrm{cm}^{2}$ in the filamentation regime, the ionization process is dominated by the multiple photoionization mechanism. The electron density can thus be obtained by

$$
\rho(\vec{r}, t)=\int_{-\infty}^{t} \sigma_{8} \rho_{\text {mol }} I^{8}\left(\vec{r}, t^{\prime}\right) d t^{\prime},
$$

where $\sigma_{8}$ and $\rho_{\mathrm{mol}}$ are the multiphoto-ionization cross section and the density of neutral oxygen molecules respectively [1]. However, when the two beams present a frequency difference, this interference pattern moves in the direction $\Delta \vec{k}=\vec{k}_{1}-\vec{k}_{2}=\Delta k \cdot \vec{u}$ with a velocity $\vec{v}_{0}=(\Delta \omega / \Delta k) \cdot \vec{u}=\left[\left(\omega_{1}-\omega_{2}\right) /\left(k_{1}-k_{2}\right)\right] \cdot \vec{u}$, where $\omega_{i}$ and $k_{i}=\omega_{i} / c$ are the instantaneous frequencies and the wave vector of the two interacting pulses and $\vec{u}$ is a unitary vector [14]. For two delayed pulses with the same chirp parameter $C$ and pulse duration $\tau_{p}$, the frequency difference can be expressed as $\Delta \omega=-C \tau_{d} / \tau_{p}^{2}$, where $\tau_{d}$ is the time delay between the two pulses $[14,15]$. Note a spelling mistake in the same equation in Ref. [14] where a number 2 appears in the denominator.

In order to observe the traveling plasma grating, we imaged the pump beams overlap region on a CCD camera, using a $20 \times$ magnification microscope objective. When the two pump pulses overlap in time, a bright plasma spark appears in air. Photographs of the bright plasma are presented in Fig. 2 for two different crossing angles. A plasma fringes structure is observed, similar to those obtained for a stationary plasma grating $[10,11]$. Measured periods of $4.46 \mu \mathrm{m}$ for $\varphi=10^{\circ}$, and $6.57 \mu \mathrm{m}$ for $6.9^{\circ}$, are in good agreement with the corresponding optical field interference periods $\Lambda$ of $4.58 \mu \mathrm{m}$ and $6.65 \mu \mathrm{m}$. The fact that fringes appear distinctly in the time integrated photographs indicates that the net displacement of the grating is much less than the interfringe period, since otherwise the fringe structure would be washed out. 
To monitor the spatial motion of this plasma grating and determine its velocity, we employed a probe pulse at $400 \mathrm{~nm}$ (see Fig. 1). The principle is the following. For a refractive index grating with period $\Lambda$, the Bragg condition reads as $n \lambda=2 \Lambda \sin (\theta)$ with $\lambda$ the wavelength, $\theta$ the scattering angle and $n$ an integer. The $400 \mathrm{~nm}$ probe beam satisfies the Bragg condition for second-order scattering if it crosses the grating collinearly to one of the pump. In another word, in the presence of the plasma grating the $400 \mathrm{~nm}$ probe propagating in the direction of pump 2 should be partially diffracted into the direction of pump 1, so in our case $\theta=\varphi / 2$. With a plasma grating moving with velocity $v_{\text {grating }}$ in the direction of $\Delta \vec{k}$, due to the Doppler effect the frequency of the diffracted probe is expected to be shifted by an amount of

$$
\Delta f=\left(v_{\text {grating }} \sin (\varphi / 2) / c\right) f_{0},
$$

where $f_{0}$ is the central frequency of the incident probe pulse. The plasma grating moves due to its excitation source, the traveling laser interference. Assuming $v_{\text {grating }} \approx v_{0}$, then the probe wavelength shift can be obtained as

$$
\Delta \lambda=\lambda_{0} C \tau_{d} / \pi f_{0} \tau_{p}^{2}
$$

where $\lambda_{0}$ is the wavelength of the incident probe pulse. Therefore, by examining the Doppler shift of the diffracted signal, it is possible to observe the motion of the grating. Experimentally we recorded the shift of the spectral gravity center of the broadband 400 $\mathrm{nm}$ signal pulse for quantitative analysis.

Measurements were performed in Argon. An atomic gas was chosen to avoid molecular alignment effect, which has a significant influence on the spectrum of an ensuing probe pulse [16]. First, the pump and probe pulses were set to be Fourier transform limited in the interaction area, by carefully compensating all the positive dispersion experienced by the pulses along the propagation (lens, half-wave plate, windows, $5 \mathrm{~m}$ of air) with an acousto-optic phase modulator in the laser. In Fig. 3(a), the gravity center of the $400 \mathrm{~nm}$ signal spectrum is presented as a function of the delay $\tau_{d}$, for $\tau_{p r-g}=0$. $\tau_{p r-g}$ equal to zero corresponds to the time when the probe beam arrives at the maximum overlap of the two pump pulses. Also, the spectrum of the probe at three different times delay $\tau_{d}=-66,0,66$ fs is presented in Fig. 3(b). No spectrum shift is observed, which confirms that the velocity of the grating is zero since $\Delta \omega=0$.

Second, the two pump pulses were negatively chirped to $\sim 91.7 \mathrm{fs}$, while the probe pulse was maintained to $40 \mathrm{fs}$. The spectral gravity center of the probe is presented in Fig. 3(c) (square dot) and three typical spectrums are shown in Fig. 3(d). It is seen that the spectrum shifts gradually with $\tau_{d}$, reflecting the motion of the plasma grating. Based on equations (2) and (3), we determined the velocity of the plasma grating and calculated the corresponding Doppler shift. The results are also presented in Fig. 3(c) (solid line). From this measurement we can extract the velocity of the grating, we find $v_{\text {grating }}=-2.3 \times 10^{7} \mathrm{~m} . \mathrm{s}^{-1}$ for $\tau_{d}=-100 \mathrm{fs}$. This is to be compared with the calculated value $v_{0}=-2.76 \times 10^{7} \mathrm{~m} \cdot \mathrm{s}^{-1}$. The velocity of the grating could be changed from $v_{\text {grating }}=-2.3 \times 10^{7} \mathrm{~m} \cdot \mathrm{s}^{-1}$ to $3.1 \times 10^{7} \mathrm{~m} \cdot \mathrm{s}^{-1}$ by changing the delay (the frequency difference) 
between the two pump pulses. The experimental results are in fair agreement with our model.

Furthermore, the simulations concerning the formation of the moving grating presented in reference [14] indicate that the grating should be at rest shortly after the passage of the pump pulses. Therefore, a delayed probe pulse should experience no frequency shift. Experimentally, we set the probe delay to $\tau_{p r-g}=1 \mathrm{ps}$ and varied the relative delay between the two pumps $\tau_{d}$ by a few hundreds of femtoseconds. The results are presented in Fig. 3 (c) (triangle). As expected, the spectrum does not present any shift.

Finally, we determined the effective duration of the grating motion. To this end, the delay between the two pumps was set to a typical value $\tau_{d}=80 \mathrm{fs}$ while the delay between the probe and the grating, $\tau_{p r-g}$, was varied. The results are presented in Fig. 4 . The Doppler shift is observed for a few tens of femtoseconds, which corresponds to the overlapping time of the two pump pulses. This shows that the grating becomes at rest within less than $100 \mathrm{fs}$.

In conclusion, the spatial motion of a dynamic plasma grating formed by two interacting filaments in gases has been characterized experimentally using the Doppler effect. It is confirmed that the traveling characteristic of the plasma grating occurs only during its generation process due to the moving excitation source, while after it remains stationary for a much longer time. The calculated results reproduce well our observations.

\section{References:}

[1] A. Couairon and A. Mysyrowicz, Phys. Rep. 441, 47 (2007).

[2] S. L. Chin, S. A. Hosseinni, W. Liu, Q. Luo, F. Théberge, N. Aközbeck, A. Becker, V.P. Kandidov, O.G. Kosareva, and H. Schroeder, Can. J. Phys. 83, 863 (2005).

[3] J. Kasparian, M. Rodriguez, G. Méjean, J. Yu, E. Salmon, H. Wille, R. Bourayou, S. Frey, Y.-B. André, A. Mysyrowicz, R. Sauerbrey, J.-P. Wolf, and L. Wöeste, Science 301, 61 (2003).

[4] A. Couairon, M. Franco, A. Mysyrowicz, J. Biegert, and U. Keller, Opt. Lett. 30, 2657 (2005).

[5] M. Rodriguez, R. Sauerbrey, H. Wille, L. Woëste, T. Fujii, Y.-B. André, A. Mysyrowicz, L. Klingbeil, K. Rethmeir, W. Kalkner, J. Kasparian, E. Salmon, J. Yu, and J.-P. Wolf, Opt. Lett. 27, 772 (2002).

[6] C. D'Amico, A. Houard, M. Franco, B. Prade, A. Mysyrowicz, A. Couairon, and V.T. Tikhonchuk, Phys. Rev. Lett. 98, 235002 (2007).

[7] T. T. Xi, X. Lu, and J. Zhang, Phys. Rev. Lett. 96, 025003 (2006).

[8] S. Suntsov, D. Abdollahpour, D. Papazoglou, and S. Tzortzakis, Opt. Express 17, 3190 (2009).

[9] X. Yang, J. Wu, Y. Tong, L. Ding, Z. Xu, and H. Zeng, Appl. Phys. Lett. 97, 071108 (2010).

[10] S. Suntsov, D. Abdollahpour, D. Papazoglou, and S. Tzortzakis, Appl. Phys. Lett. 94, 251104 (2009).

[11] X. Yang, J. Wu, Y. Peng, Y. Tong, P. Lu, L. Ding, Z. Xu, and H. Zeng, Opt. Lett. 24, 3806 (2009).

[12] A. C. Berstein, M. McCormick, G.M. Dyer, J.C. Sanders, and T. Ditmire, Phys. Rev. Lett. 102, 123902 (2009).

[13] B. Shim, S. Schrauth, C.J. Hensley, L. T. Vuong, P. Hui, A. A. Ishaaya, and A. L. Gaeta, Phys. Rev. A 81, 061803 (2010).

[14] Y. Liu, M. Durand, S. Chen. A. Houard, B. Prade, and A. Mysyrowicz, Phys. Rev. Lett. 105, 055003 (2010).

[15] S. Smolorz, and F. Wise, J. Opt. Soc. Am. B 17, 1636 (2000).

[16] S. Varma, Y.-H. Chen, and H. M. Milchberg, Phys. Rev. Lett. 101, 205001 (2008). 


\section{Figures}

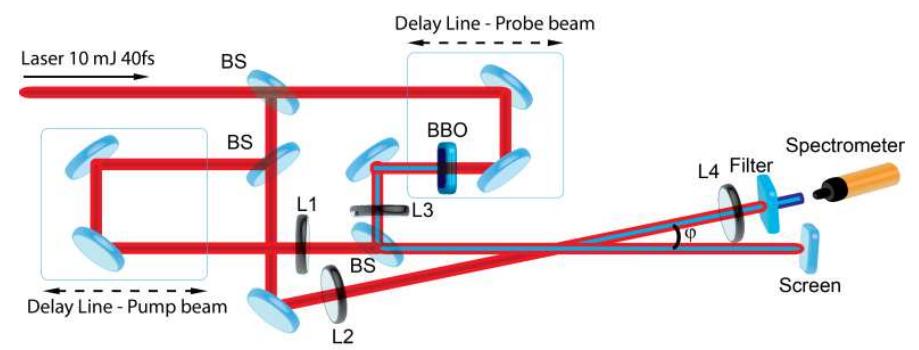

Fig. 1. Schematic experimental setup.

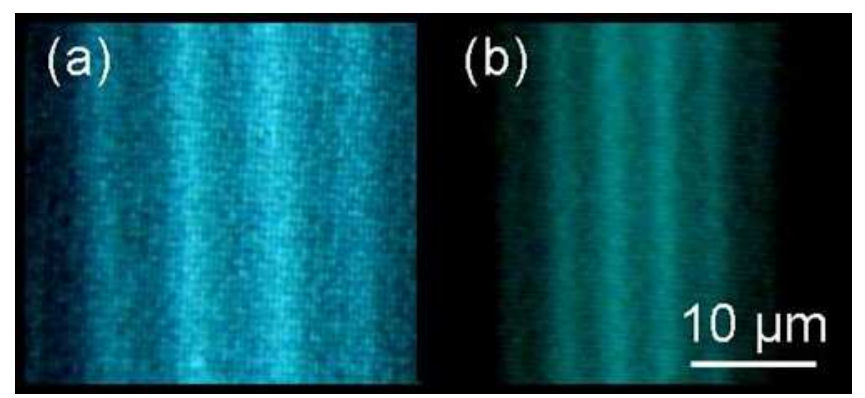

Fig. 2. Images of the plasma grating induced in air by two crossing filaments. The crossing angle of the two filaments is $6.9^{\circ}$ in (a) and $10^{\circ}$ in (b).

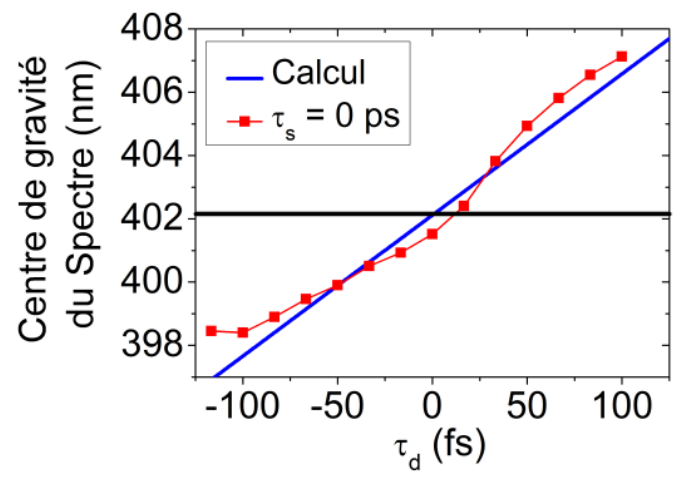

Fig. 3. Spectral gravity center of the $400 \mathrm{~nm}$ probe pulse detected in the direction of pulse 1 as a function of the delay between the two pumps $\tau_{d}$ in Argon: (a) for pump pulse of 40 fs $(\Delta \omega=0)$, (c) for pump pulses negatively chirped to $91.7 \mathrm{fs}$. The corresponding spectra of the $400 \mathrm{~nm}$ signal at different delays $\tau_{d}$ are respectively presented in (b) and (d) for the cases (a) and (c). In (c) the triangles stand for the case $\tau_{p r-g}=1 \mathrm{ps}$, the square for $\tau_{p r-g}=0$ ps. The solid line shows the calculated results based on equation (3) for $\tau_{p r-g}=0$ ps. 


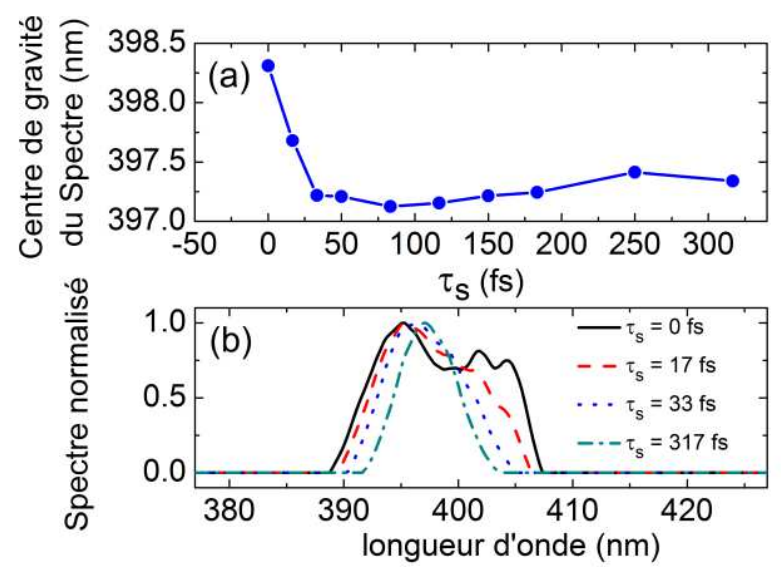

Fig. 4. Spectral gravity center (a) and normalized spectrum (b) of the $400 \mathrm{~nm}$ signal as a function of the delay between the probe and the grating, $\tau_{p r-g}$. The pump pulses delayed by $\tau_{d}=80 \mathrm{fs}$, are negatively chirped to $91.7 \mathrm{fs}$. 